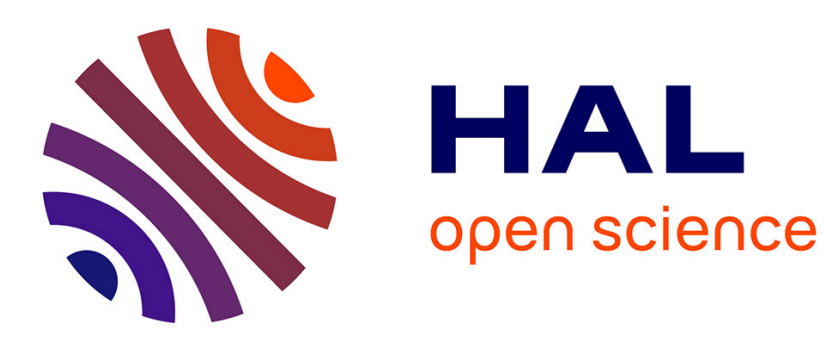

\title{
Sociologie du développement ou développement de la sociologie: la question des femmes et de leur travail
}

\author{
Anne-Marie Daune-Richard
}

\section{To cite this version:}

Anne-Marie Daune-Richard. Sociologie du développement ou développement de la sociologie: la question des femmes et de leur travail. Revue Tiers Monde, 1982, XXIII (90), pp.375-387. hal03508750

\section{HAL Id: hal-03508750 \\ https://hal.science/hal-03508750}

Submitted on 24 Jan 2022

HAL is a multi-disciplinary open access archive for the deposit and dissemination of scientific research documents, whether they are published or not. The documents may come from teaching and research institutions in France or abroad, or from public or private research centers.
L'archive ouverte pluridisciplinaire HAL, est destinée au dépôt et à la diffusion de documents scientifiques de niveau recherche, publiés ou non, émanant des établissements d'enseignement et de recherche français ou étrangers, des laboratoires publics ou privés. 


\section{Sociologie du développement ou développement de la sociologie} : la question des femmes et de leur travail

Anne-Marie Daune-Richard

\section{Citer ce document / Cite this document :}

Daune-Richard Anne-Marie. Sociologie du développement ou développement de la sociologie : la question des femmes et de leur travail. In: Tiers-Monde, tome 23, n90, 1982. Sociologie du développement. pp. 375-387;

doi : https://doi.org/10.3406/tiers.1982.4126

https://www.persee.fr/doc/tiers_0040-7356_1982_num_23_90_4126

Fichier pdf généré le 01/04/2019 


\title{
SOCIOLOGIE DU DÉVELOPPEMENT OU DÉVELOPPEMENT DE LA SOCIOLOGIE : LA QUESTION DES FEMMES ET DE LEUR TRAVAIL
}

\author{
par Anne-Marie Daune-Richard*
}

\begin{abstract}
Ayant précédemment développé une réflexion sur le travail des femmes dans les pays en voie de développement, j'ai été amenée à poursuivre une recherche sur le même thème dans un contexte sociologique résolument éloigné : celui d'une grande ville française. Or mon expérience antérieure, loin de me laisser démunie devant des questions radicalement nouvelles, a constitué au contraire un appui théorique et méthodologique de première importance. Bien plus, c'est d'une approche de ces questions à partir d'une réflexion sur les PVD que sont nées mes préoccupations concernant les pays du Centre. Un tel itinéraire s'inscrit fondamentalement dans un mouvement de compréhension des processus de développement des sociétés, en particulier sous l'effet de l'industrialisation progressive des économies et ce, tant au centre qu'à la périphérie. Pour suivre cet itinéraire, l'article ci-dessous comprendra deux parties : l'une sera consacrée à la question du travail des femmes dans les processus de développement, l'autre essaiera de montrer la proximité des problèmes théoriques et méthodologiques lorsque l'on essaie d'examiner cette question dans un pays comme la France.
\end{abstract}

\section{I. - La question du TRAvail des femmes DANS LES PROCESSUS DE DÉVELOPPEMENT}

Au cours des dernières décennies, les travaux de nombreux chercheurs, économistes et sociologues, du monde entier ont permis une meilleure compréhension des grandes mutations sociales, économiques et

* Attachée de recherche au cNRs.

Revue Tiers Monde, t. XXIII, no 90, Avril-Juin 1982 
politiques qu'ont subies les pays en voie de développement dans leur intégration progressive au marché international, à travers les différentes étapes de la colonisation et de l'expansion du capitalisme. En même temps l'analyse des formes qu'ont pu revêtir, pour les femmes, ces grands bouleversements sociaux restait très embryonnaire. Cependant, dans un passé très récent, des tentatives ont été menées (en particulier aux Etats-Unis mais aussi en France) pour coordonner et exploiter des connaissances qui, sur ce sujet, étaient le plus souvent fragmentaires et surtout très dispersées. Ainsi se dessinent les grandes lignes d'une réflexion sur les changements qui affectent tant les modes de vie que les rôles économiques et sociaux des femmes dans les sociétés dites « en voie de développement ».

Les transformations sociales qui ont affecté ces sociétés sont fondamentalement liées à l'introduction forcée - plus ou moins brutale selon les époques et les lieux - d'une économie marchande, dont le caractère dépendant a constitué la base même du développement et de l'expansion capitaliste. Ainsi s'est opérée une mutation dans les finalités des systèmes économiques qui a eu une influence directe sur les formes d'organisation sociale de la production et, en particulier, sur l'affectation de la force de travail. C'est à ce niveau que, de notre point de vue, se situent les éléments moteurs qui ont guidé l'évolution de la place et du rôle social des femmes dans ces pays.

Dans les sociétés précapitalistes, en effet, l'essentiel de la production n'est pas destiné à la vente mais à la subsistance. Cela ne veut pas dire qu'il n'existe pas de circuits d'échanges, mais ceux-ci ne concernent que les surplus; en tout état de cause, il n'existe pas de structures de production spécialisées pour la vente.

La division du travail y est extrêmement normalisée et directement liée aux structures de la parenté. Pour ce qui concerne les catégories de sexe, la division sexuelle du travail est très présente et revêt une signification toute autre - quant à la place sociale des femmes - que celle que nous connaissons actuellement. En effet, si les femmes sont exclues de certains processus de production précis - comme, par exemple, le plus souvent, la chasse, le travail du fer et celui du bois ${ }^{\mathbf{p}}$ - il n'en reste pas moins que toutes les femmes participent largement à la production agricole, dominante dans des sociétés majoritairement agraires, et à certaines activités artisanales. De plus, dans de nombreux domaines de l'activité - en particulier l'agriculture et l'habitat - la division sexuelle du travail définit une répartition des tâches selon les différentes étapes

x. Paola TABEt, Les mains, les outils, les armes, in L'Homme, t. XIX, no 3-4, juilletdécembre 1979. 
d'un même processus de production. Par exemple, ce sont les hommes qui vont défricher et labourer, les femmes semer et désherber, les hommes et les femmes ensemble qui vont assurer la récolte puis les femmes qui seront chargées du transport, etc. La répartition du travail entre les sexes laisse donc aux femmes un rôle important dans la poursuite des activités qui assurent l'essentiel de la subsistance de ces sociétés. Bien plus, dans de nombreuses ethnies d'Amérique du Sud et d'Afrique, les femmes avaient - et ont encore parfois - accès à des champs personnels qu'elles travaillaient elles-mêmes et dont le produit leur revenait. Il en allait de même pour les activités artisanales dans lesquelles elles pouvaient être spécialisées.

Parallèlement, l'activité domestique des femmes, si elle s'inscrit bien dans la division des tâches entre sexes, ne constitue pas un secteur marginalisé de la production sociale. En effet, le travail domestique (au sens propre de : lié à la maison) comprend généralement une part importante de la production agricole (qui se trouve encore aujourd'hui en Afrique de l'Ouest dans les « champs de cases ») et/ou artisanale : poterie, tissage. Un autre volet du travail domestique féminin est la transformation des produits alimentaires pour leur consommation et leur conservation : tâches astreignantes mais faisant appel à un réel savoir technique, indispensables à la survie du groupe en l'absence de produits de remplacement. Dans ce cadre s'inscrit aussi la dure collecte quotidienne du combustible et de l'eau.

Pour les tâches plus directement liées à la famille, et en particulier à l'éducation des enfants, il faut noter que, loin d'être toujours concentrées sur la mère, elles sont largement socialisées et sont le fait - comme l'indiquent les termes mêmes de la parenté classificatoire - de l'ensemble des membres de la communauté. Paradoxalement, ces sociétés dans lesquelles la fonction maternelle, en tant que fonction de reproduction, est si fondamentale que l'essentiel des relations interethniques correspond à des circuits d'échanges matrimoniaux, développent donc des modèles de comportements féminins beaucoup moins spécialisés dans un rôle exclusivement maternel qu'ils ne le sont dans les sociétés actuelles.

Contrairement à ce que nous connaissons dans les sociétés industrielles, la plupart des sociétés précapitalistes (les exceptions sont toutes le fait de sociétés étatisées et bureaucratisées) n'établissaient donc pas, pour les femmes, de frontière entre leurs rôles de mère et de travailleuseproductrice. D'une part, toutes étaient à la fois mères et productrices; d'autre part, l'autonomisation relative qui s'est établie, avec le développement du capitalisme, entre la production et la reproduction ne pouvait s'opérer dans ces sociétés du fait de l'inexistence d'un secteur'marchand. 
Il existait toutefois deux sphères distinctes et hiérarchisées de l'organisation sociale : celle des biens de subsistance et celle des biens de prestige, qui différaient tant par le mode de fonctionnement et la nature des produits que par les acteurs sociaux en jeu. Ces biens de prestige étaient socialement limités et contrôlés, et uniquement destinés à la circulation; l'accès à ces biens était l'expression même du pouvoir social. Or, c'est à ce niveau que se situent, pour les femmes des sociétés précapitalistes, les limites du champ de leur action - et en même temps de leur pouvoir - social; en effet, elles étaient par définition exclues de l'accès à ces biens de prestige puisque la fonction première de ceux-ci était de jouer le rôle de convention créancière dans les échanges matrimoniaux ${ }^{2}$. Dans ce contexte, c'était la rigidité des rôles sociaux - et en particulier celle des rôles sexuels - qui garantissait le consensus social, plus que la différenciation des secteurs de production, ce qui est le cas dans les sociétés industrialisées.

Les rôles sexuels étaient donc socialement délimités et la rigidité de leur définition établissait une coupure très nette entre le groupe des femmes, d'un côté, celui des hommes, de l'autre ; les normes régissant leurs fonctionnements respectifs et leurs relations (ainsi que les transgressions possibles) étaient précisément codifiées. Cela favorisait bien sûr une identification sociale plus claire des individus mais développait en outre, au sein de ces deux groupes, des modes de fonctionnement très socialisés. Les formes plus anciennes et plus radicales de la colonisation en Amérique latine ont considérablement affaibli ces modes de vie traditionnels, mais dans toutes les campagnes africaines on rencontre encore aujourd'hui ces groupes de femmes réunies pour les corvées d'eau ou de bois, la lessive, le pillage ou le décorticage des céréales ou encore... la coiffure.

Ainsi, en terme de pouvoir social, les femmes des sociétés précapitalistes ont toujours été - à des degrés divers - insérées dans des rapports de domination masculine et, pratiquement toujours, exclues des responsabilités politiques. Mais l'importance et la diversité de leurs tâches ainsi que la participation de toutes aux principales activités économiques leur faisaient jouer un rôle social à part entière. D'autant plus que le haut degré de socialisation de leurs activités leur permettait d'avoir une réelle conscience de leur place - et de leur poids - en tant que groupe social, quelles que soient par ailleurs les difficultés matérielles de leurs conditions de vie.

Le développement et la modernisation ont été (et sont) le plus souvent considérés comme des facteurs décisifs d'amélioration de la vie des

2. Cf. les travaux d'anthropologues comme Raymond Firth, Maurice Godelier. 
individus dans les pays du Tiers Monde. Qu'en est-il pour les femmes de ces pays?

Le développement a d'abord signifié pour les populations soumises à l'expansion européenne l'obligation de fournir puis exploiter des matières premières - minières et/ou agricoles - destinées à l'exportation. Ce bouleversement dans les finalités mêmes de l'activité économique a profondément transformé l'organisation de la production. Pour les femmes, l'élément décisif du changement a été la différenciation progressive de l'économie en deux secteurs : celui de la production marchande, d'une part, celui de la production vivrière, d'autre part. Dans ce processus les hommes ont été contraints - par tous les moyens dont ont pu se doter les colonisateurs - de travailler pour le secteur marchand, les femmes devant assurer l'essentiel de la production vivrière. En Amérique latine, les formes d'implantation des colons dans de grands domaines agraires témoignent de cette réorganisation de la force de travail : les hommes reçoivent, en échange d'une partie de leur travail dans les plantations, des lopins de terre qui sont principalement cultivés par les femmes et sur lesquels ils donnent un coup de main quand ils le peuvent. En Afrique, ce sont les grandes migrations, d'abord liées à l'esclavage et au travail forcé, puis - avec la désorganistaion des sociétés traditionnelles - à la nécessité économique, qui laissaient aux femmes la charge d'assurer la subsistance des communautés rurales avec l'aide des enfants et des vieillards. Dans ce contexte, elles ont été amenées à développer leurs responsabilités dans l'agriculture, mais aussi leur savoir-faire artisanal et commercial.

Après ces premières étapes marquées par une forte déstructuration sociale, les formes de la colonisation ont permis une stabilisation de la situation et des populations; les contraintes économiques (en particulier l'impôt) se substituent - en partie tout au moins - à la force brutale pour inciter les hommes à s'insérer dans la production marchande. Dans tous les continents, les catégories de la pensée occidentale s'imposent : pour les femmes, les notions de propriété privée et de chef de famille ont pour corollaire la progressive non-reconnaissance sociale de leur travail.

En effet, l'accession à la propriété concerne les hommes qui, juridiquement, deviennent chefs de famille. Ainsi, alors que leurs charges de travail se sont accrues, les femmes voient leurs droits et la relative autonomie personnelle dont elles jouissaient se restreindre : dans ce nouveau cadre juridique, elles deviennent dépendantes de leurs maris et/ou de leurs pères. Dans le continent latino-américain où le contexte historique et les formes de colonisation ont permis une pénétration massive de la 
religion catholique, celle-ci a eu une influence déterminante pour asseoir le nouveau statut des femmes.

Avec le développement des techniques, la marginalisation des femmes prend un tournant décisif : les programmes de modernisation rurales, les réformes agraires, la mise en place de coopératives visent à améliorer la productivité et les circuits de commercialisation des cultures marchandes et s'adressent donc prioritairement aux hommes. Ainsi, non seulement les femmes perdent-elles tout contrôle sur la terre mais elles se voient exclues et de la formation technique et de l'accès aux circuits de crédit et de commercialisation. Quand les services de développement rural s'adressent à elles, ce sont des cours de couture, d'hygiène ou de diététique qui leur sont proposés. Dans ces conditions, ne nous étonnons pas que « le développement ait signifié globalement, pour les pays du Tiers Monde, baisse de la production et de la productivité des cultures vivrières ${ }^{3}$.

Ainsi arrive-t-on à cette situation paradoxale : en Afrique, par exemple, et d'après l'Organisation des Etats africains, 60 à $80 \%$ de la production vivrière serait assurée par les femmes - en dehors des circuits de la production marchande - alors que, selon un document du ministère du Travail américain ${ }^{4}$, seules $5 \%$ des femmes de ce continent « travaillent ".

Sous l'effet conjugué de la déstabilisation sociale et de la paupérisation des campagnes (détérioration des termes de l'échange et déclin de la production vivrière), l'exode rural prend une ampleur inquiétante : nombreux sont les hommes et les femmes qui prennent le chemin de la ville en quête d'un mieux vivre. Pour les femmes, l'alourdissement de leurs tâches et la dégradation relative des conditions de leur vie sociale rendent le mirage de la vie urbaine plus tentant encore que pour les hommes. Or, pour la majorité d'entre elles, leur insertion s'y fait dans des conditions extrêmement précaires. Sous-qualifiées, leurs chances de trouver un emploi stable et correctement rémunéré sont quasi nulles : au mieux, elles arrivent à se placer dans les services domestiques ou comme vendeuses, mais plus généralement elles survivent grâce à de petites activités marchandes ou à la prostitution.

Même dans les pays du Tiers Monde connaissant une plus longue tradition urbaine et industrielle, le pourcentage des femmes recensées

3. Cf. Esther Boserup, Woman's role in economic development, London, Georges Allen \& Unwin ltd., 1970.

4. Cité in Irene TrNkER, The adverse impact of development on women, in Irene TINKER and Michèle Bo Bramsen, Women and world developement, Washington, Overseas Development Council, 1975. 
comme " économiquement actives " reste faible (environ $30 \%$ pour le Brésil); et encore les trouve-t-on majoritairement dans des emplois sous-qualifiés du primaire ou du tertiaire.

Parallèlement, l'insertion des hommes sur le marché de l'emploi est très problématique : dans la plupart des villes du Tiers Monde, l'importance du chômage et des bas salaires va en s'aggravant Les migrations de main-d'œuvre en quête d'emploi et l'insuffisance des revenus masculins ont souvent laissé aux femmes la responsabilité de subvenir aux besoins de la famille, alors même que les solidarités inhérentes à la traditionnelle réciprocité des relations de parenté s'affaiblissaient ou même disparaissaient. Ce processus entamé depuis bien longtemps en Amérique latine éclaire sans doute le fait que dans certaines parties de ce continent $50 \%$ des ménages ont une femme comme chef de famille ${ }^{5}$. En Afrique, et dans une moindre mesure en Asie, où les colonisations ont perpétué plus longtemps (pour des raisons qui n'entrent pas directement dans le cadre de cette étude) des traditions, même dénaturées, d'une vie communautaire, les relations de parenté sont demeurées très vivaces jusque dans un passé técent.

Or, le processus de paupérisation qui affecte ce continent s'est considérablement aggravé dans la dernière décennie; dans ce contexte, les femmes en milieu urbain - mais aussi les femmes en milieu rural ont tendance à vouloir augmenter à tout prix leurs revenus personnels, afin de mieux assurer leur avenir en développant leurs propres réseaux de solidaritéb. Ainsi assiste-t-on actuellement à une très forte déstabilisation des rôles familiaux et, en particulier, à une augmentation des femmes « chefs de famille » qui peuvent même se regrouper en quartiers (comme celui des brasseuses de bière dans la banlieue de Nairobi) ${ }^{\text {. }}$.

Dans les toutes dernières années on remarque une nouvelle orientation des grandes organisations internationales qui semblent vouloir développer des programmes en faveur de la promotion de la femme, en particulier dans le domaine de l'emploi (BIT) mais aussi dans celui de l'éducation (Unesco). S'il est encore trop tôt pour dresser un premier bilan d'actions qui sont à peine engagées ${ }^{8}$ il est important, pour saisir quelles tendances peut prendre cette promotion des femmes du Tiers

5. Cf. Irene Tinker, op. cit.

6. Cf. Claudine VIDAL, Guerre des sexes à Adibjan. Masculin, féminin, CFA, in Cabiers d'Etudes africaines, vol. XVII, no 65, 1977 .

7. Nici Nelson, Survivre dans un milieu hostile, in CERES, vol. 8, $\mathrm{n}^{\circ} \mathbf{2}$, mars-avril 1975.

8. Yvonne Mignot-Lefèbvre, "Femmes et développement : les nouvelles stratégies des organisations internationales ». Communication faite a la Table-Ronde sur «Les femmes, la division internationale du travail et le développement », organisée par Mme Andrée MicheL, maître de recherche au CNRS, Paris, mai 1980. 
Monde dans les prochaines décennies, de situer cette réorientation dans son contexte international.

La recrudescence de graves famines dans l'ensemble de ces pays au cours des années 70 a dramatiquement focalisé l'attention sur le problème structurel du secteur vivrier. De nombreux Etats (en particulier en Afrique, où cette question concerne - à des degrés divers - l'ensemble du continent), soutenus par l'aide internationale, s'efforcent d'élargir la production vivrière et d'en améliorer la productivité. Cette tendance devrait permettre aux femmes rurales, qui participent majoritairement au secteur vivrier, d'espérer connaître bientôt de meilleures conditions de vie et de travail. D'ores et déjà des mesures institutionnelles ont été prises dans certains pays permettant l'accès des femmes aux circuits de commercialisation et de crédit (coopératives) ou même (comme au Sénégal) aux intances politiques du développement régional. Malheureusement, ces possibilités sont loin, pour l'instant, d'être pleinement utilisées par les intéressées...

Mais ce soudain intérêt pour la promotion des femmes du Tiers Monde s'inscrit aussi dans le contexte de la nouvelle division internationale du travail qui spécialise les pays développés du Centre dans les industries à technologies de pointe et à forte intensité de capital et dans la vente de sa "matière grise " sous forme de brevets, d'usines clefs en main, tandis que dans le même temps les industries à technologie courante et forte intensité de main-d'œuvre (non qualifiée) se délocalisent dans les PVD.

Dans ce contexte, l'Amérique latine depuis déjà longtemps constitue plus un marché pour les produits européens - et en particulier la technologie - qu'un réservoir de main-d'œuvre, rôle précédemment réservé au continent asiatique dans les limites des changements politiques qui l'ont affecté. La plus grande partie du continent africain offre par contre un champ relativement vierge pour l'extension de cette nouvelle division internationale du travail : la crise du secteur agro-exportateur, les efforts de relance du secteur vivrier, le blocage par la plupart des pays européens des flux d'immigration qui s'étaient mis en place depuis l'ère coloniale laissent présager pour ce continent, dans les années qui viennent, de grands mouvements de main-d'œuvre.

Quelles perspectives cette évolution peut-elle offrir aux femmes? Il est à craindre que l'avenir ne s'ouvre pas pour elles sous les meilleurs auspices, malgré la proclamation par les Nations Unies, en 1975, d'une «Décade de la femme».

En effet, l'expérience des pays déjà industrialisés d'Amérique latine montre que l'ère des multinationales n'est pas forcément créatrice 
d'emploi, ni ne conduit nécessairement à une amélioration du niveau de vie. Ainsi, au Brésil, les salaires réels ont baissé de 30 à $40 \%$ depuis 1964. Par ailleurs, l'installation d'une entreprise moderne dans un secteur déterminé, si elle crée des emplois, développe en même temps - dans la plupart des cas - un chômage encore plus grand, en éliminant du marché les petits artisans locaux travaillant dans le même secteur.

Pour les femmes, déjà largement marginalisées par rapport au marché de l'emploi, l'aggravation du chômage et les restrictions touchant le secteur de la petite production marchande ne peuvent que les atteindre de plein fouet. En effet, dans de nombreux endroits, des femmes ont réussi à constituer et développer des marchés qu'elles contrôlent elles-mêmes, pour écouler leurs propres productions, tant dans le tissage, la préparation des vêtements ou la poterie que dans les activités plus spécifiques comme la préparation de l'huile (Nigeria) ou le brassage de la bière. Parfois les réseaux qu'elles mettent en place pour approvisionner les marchés concurrencent purement et simplement des circuits modernes de commercialisation, qu'ils soient étatiques (comme à Yaoundé) ou privés (comme à Managua). Il faudrait évidemment parler du prix payé par ces femmes pour arriver à de tels résultats, en termes de mode de vie...

Finalement, dans les tendances actuelles du « développement ", une double perspective semble s'ouvrir pour les femmes :

- pour les femmes rurales, la dégradation des cultures marchandes et la mécanisation des cultures vivrières risquent de les mettre en concurrence avec les hommes. On sait en effet que de tout temps, mais en particulier avec la division établie au cours du développement capitaliste, entre la production pour le marché et les tâches liées à la reproduction, la mécanisation d'une activité est prioritairement appropriée par les hommes;

- le développement d'une industrialisation - de nature dépendante offrira sans doute pour les femmes des emplois dans l'industrie (conserveries, textile et habillement, électronique) et dans le tertiaire. Mais l'aggravation de la situation de l'emploi risque de les toucher au premier chef, tant sur le plan du chômage que sur celui du niveau des salaires. De plus, même pour celles qui occupent des emplois relativement qualifiés, leur accès à des secteurs traditionnellement féminisés - comme ceux de l'enseignement et de la santé - risque d'être progressivement réduit par la concurrence sur le plan de l'emploi et par le développement de modèles culturels moins rigides quant à la ségrégation sexuelle. 
Les femmes du Tiers Monde se sont souvent opposées à ce processus, mais de façon dispersée : les nouvelles formes de leur insertion sociale risquent de donner une dimension plus «politique » à des luttes qui ont majoritairement conservé un impact local. L'organisation de « coordinations " ou de mouvements de femmes dans les différents pays et continents est l'un des aspects de cette évolution. Les données historiques de la question « Femmes et développement " s'en trouveront probablement modifiées dans un avenir proche.

\section{II. - LA PROXIMITÉ DES PROBLÈMES THÉORIQUES ET MÉTHODOLOGIQUES}

Si l'on observe l'évolution de la place du travail des femmes du Tiers Monde dans les processus de développement et les incidences qu'elle peut avoir sur la nature de leur rôle social, il n'apparaît pas évident, nous l'avons vu, que celui-ci soit massivement étendu et renforcé par un développement pourtant souvent tenu pour synonyme de progrès. C'est en partant d'une telle constatation que j'ai ressenti la nécessité d'examiner de plus près la question du travail des femmes telle qu'elle se présente dans le contexte de progrès technique propre à un pays "développé ». Or, dans cette démarche, les difficultés rencontrées m'ont semblé étrangement proches tant au niveau théorique qu'au niveau méthodologique.

I) Au niveau théorique la pesanteur idéologique des concepts de développement et/ou de progrès est si considérable qu'il occulte purement et simplement certaines données statistiques pourtant connues. Prenons deux exemples précis : il est socialement connu que les femmes françaises d'aujourd'hui " travaillent " plus souvent que celles des générations précédentes. Effectivement entre les deux recensements de 1968 et de 1975 on constate une élévation considérable des taux d'activité féminins, en particulier chez les femmes mariées de moins de 35 ans. Mais la réalité connue des observateurs - économistes ou statisticiens qui montre que si les femmes constituent $38 \%$ de la population active en 1975 , elles en représentaient déjà $37,13 \%$ en 1906 , cette réalité-là n'est guère « passée » dans les médias. Bien sûr le « travail » des femmes est à l'heure actuelle massivement constitué d'emplois salariés; mais est-ce à dire que leurs grand-mères ou arrière-grand-mères, majoritairement insérées dans les travaux agricoles, ne "travaillaient pas "? N'oublions pas d'ailleurs que les emplois féminins dans le secteur secondaire étaient 
de un peu plus de 2 millions en 1906 et sont un peu moins de 2 millions aujourd'hui, se maintenant autour de $26 \%$ de la population active féminine française ${ }^{9}$.

L'autre exemple concerne le travail domestique des femmes : si toutes les femmes ne sont pas convaincues que "Moulinex libère la femme ", au moins est-il massivement reconnu que la mécanisation des tâches ménagères a permis de diminuer significativement les temps consactés à ces différentes tâches. Or, les données recueillies dans les enquêtes de budget temps depuis le début du siècle font apparaître que les durées hebdomadaires ou journalières de travail domestique, pour les différentes catégories de femmes actives, semblent se maintenir tant en France qu'en Grande-Bretagne et aux Etats-Unis, et ce malgré le grand boum de l'électroménager et l'apparition des nouveaux matériaux (en particulier dans le textile) après la deuxième guerre mondiale ${ }^{\mathbf{1 0}}$.

2) Au niveau de la problématique, il ne m'apparaissait pas possible d'élaborer une réflexion sur le travail des femmes en France en se limitant au travail professionnel, à la question de l'emploi féminin. Il $\mathrm{m}$ 'a semblé indispensable d'interroger la notion de travail dans le double aspect qu'il peut revêtir pour les femmes selon qu'il s'inscrit dans le cadre professionnel ou domestique. En ce sens j'ai dû, pour ne pas sectorialiser l'objet de la recherche, poser une problématique en termes $d$ 'articulation entre les deux secteurs de l'activité féminine qui se sont progressivement dissociés avec le développement de l'industrialisation : celui de la production pour le marché d'une part, celui de la production non marchande et de la « reproduction » d'autre part.

Ce faisant nous touchons à la question de la frontière entre les disciplines : en effet, pour ce qui est de mon objet de recherche, si l'activité professionnelle des femmes relève de la sociologie du travail, leur activité domestique renvoie à la sociologie de la famille; les études sur le travail féminin s'étant essentiellement consacrées à établir des corrélations entre leur activité professionnelle et la poursuite de leur rôle domestique. Or, cette nécessité de globaliser les problématiques, de dépasser les frontières des disciplines, de poser les questions en termes "d'articulation " est bien l'une des caractéristiques des recherches ayant trait au développement, nécessité d'ailleurs la plupart du temps imposée par la nature même des objets et terrains de recherche.

9. Sonia B. Fernandez de Espinosa, Evolution de l'emploi féminin en France depuis le début du siècle, Bulletin d'information du Centre d'Etude de l'Emploi, avril I981.

ro. Cf. Onknex, Tbe Sociology of House work, New York, Pantheon Books, 1974. 
3) La question des méthodes et des outils. - Développant pour la première fois un terrain de recherche français et m'apprêtant à utiliser massivement les moyens lourds de recueil statistique et historique propres à un pays développé, il a fallu rapidement me rendre à l'évidence : la réalité sociale et économique " connue " n'est pas nécessairement - loin de là - le reflet de la réalité sociale "vécue », et des pans entiers de cette dernière sont purement et simplement occultés non seulement par les médias mais aussi par les scientifiques. Dans le cadre d'une recherche sur les pratiques et les représentations du travail chez les femmes et leur évolution et transmission dans les lignées féminines, on peut énumérer sommairement les «blancs » en matière de données :

- mis à part les enquêtes de budget temps, absence quasi totale dans la recherche française instituée de travaux sur le «travail domestique». L'essentiel de la littérature à ce sujet émane - et ce n'est pas un hasard - de revues féministes ou politiques;

- absence totale dans les recueils systématiques de données statistiques de l'INSEE, du ministère du Travail, de l'INED, d'informations sur les relations mères-filles concernant tant l'emploi (enquêtes $\mathrm{FQP}$ ) que les pratiques domestiques;

- concernant l'emploi féminin il n'existe pas d'étude propre sur le travail posté des femmes employées et ouvrières (forme d'organisation du travail qui s'est considérablement développée dans les dernières années avec l'automatisation des chaînes et l'informatisation de certaines tâches). En effet les études sur le travail posté concernent essentiellement les $3 \times 8$; mais le travail de nuit étant interdit pour les femmes dans ces csp..., on ne connaît pas grand-chose de la vie des femmes en $2 \times 8$;

- l'exploitation du recensement ne prévoit pas de corrélation détaillée sexe/csp/situation de famille. Autrement dit il n'est pas possible de vérifier si certaines situations de familles ne se retrouvent pas de façon massive dans certaines CSP, et ce de façon différente chez les hommes et chez les femmes. On sait déjà que chez les femmes cadres supérieurs la proportion de célibataires est largement supérieure à la moyenne nationale : il semblerait bien que dans certaines catégories d'ouvrières les femmes veuves ou divorcées soient massivement représentées;

- concernant les salaires, il n'existe pas d'informations statistiques détaillées qui pourraient permettre de comparer les salaires de l'homme et de la femme à l'intérieur des ménages. Autrement dit on peut comparer les salaires hommes/femmes en référence aux 
emplois, mais pas en telation au mariage. L'indivisibilité de la catégorie "ménage » dans la Comptabilité nationale freine ici encore considérablement la recherche sur les femmes ${ }^{11}$;

- enfin dernier handicap et ce n'est pas le moindre : il n'existe aucun moyen systématique de constituer un échantillon de femmes en fonction de leur CS, "l'entrée » des fichiers (allocations familiales par exemple) se faisant par la csp du chef de famille..., c'est-à-dire l'homme dans la majorité des cas.

Dans ces conditions on voit que, pour certains objets de recherche, les méthodes de travail s'apparentent plus à l'empirisme et au pragmatisme inévitables dans des sociétés longtemps qualifiées de " sans histoire » faute de données systématiquement recensées, qu'à celles censées garantir la scientificité des travaux de recherche dans les pays du Centre.

\section{CONCLUSION : SOCIOLOGIE DU DÉVELOPPEMENT \\ OU DÉVELOPPEMENT DE LA SOCIOLOGIE?}

A la lumière des réflexions menées plus haut il est difficile de conclure à la spécificité d'une sociologie du développement qui porterait sur des pays « en voie de développement». Il a été montré combien les problèmes posés au chercheur tant au niveau des problématiques qu'au niveau des méthodes de travail pouvaient finalement se trouver très proches. Plutôt qu'une spécificité de la sociologie du développement ne touche-t-on pas ici à un nécessaire développement de la sociologie ? L'expérience des sociologues ayant travaillé sur les problèmes du développement les a obligés - face à la complexité de questions qui mêlent constamment la synchronie et la diachronie - - à faire preuve de créativité dans leurs problématiques. Leur spécificité ne résiderait-elle pas tout simplement là ? En ce sens transgresser les frontières entre les disciplines, diversifier les champs de recherche, élargir au maximum les problématiques pour prendre en compte tous les éléments d'une question, avoir recours aux outils de travail les plus divers - bref être peu "scientifique " - pourraient créer un climat favorable à l' "invention " sociologique. Il s'agirait alors d'un développement souhaitable de la sociologie... Le débat est ouvert.

Octobre $198 \mathrm{I}$.

I I. Pour une critique de la catégorie « ménage » a propos du travail domestique des femmes cf. Ch. Delphy, Travail ménager ou travail domestique, in A. Michel et al., Les femmes dans la société marcbande, PUF, 1978. 